\title{
Assignment optimization of pandemic influenza antiviral drugs in Urban pharmacies
}

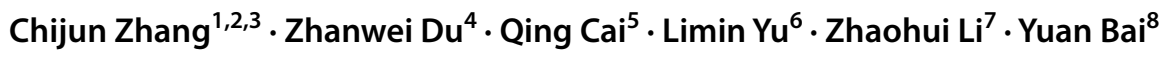

Received: 8 January 2018 / Accepted: 19 May 2018 / Published online: 1 June 2018

c) Springer-Verlag GmbH Germany, part of Springer Nature 2018

\begin{abstract}
Antiviral drugs have benefited public health officers to elucidate outbreak risks by controlling influenza pandemics efficacious, especially effective in the early stage of epidemic outbreaks. To limit explosive strain on hospitals, commercial pharmacies have joined, as antiviral drug-dispensing partners, in governments' pandemic response plans. Existing researches focus on site selection by optimizing the single objective of access to the target population. However, there are substantial inevitable but essential social factors (such as social unbalance, spatial unbalance and resource unbalance) needed to consider to benefit the society best. In this paper, we propose a network-perspective optimization model across multiple social scales (e.g, access, social unbalance, spatial unbalance and resource unbalance) to assign antiviral drugs to the urban dispensing pharmacies. In the network-based frame, we transfer these considerations to the constraints of group, edge, and node. The constrained optimization model is studied and solved using methods of willingness-to-travel model, $L_{12}$ norm and network lasso, corresponding to each considerations. Taking Shanghai in a cohort of 11 million individuals as an example, we have shown the flexibility of the proposed multi-objective model, comparing with the traditional methods. For example, we found that there are 29 pharmacies needed with covering $81 \%$ districts by tradition single-objective method. In the contrast, only 12 pharmacies are needed with similar access ability but can still cover $75 \%$ districts. Or more pharmacies are assigned with covering $87 \%$ districts. This research can supply an initial exploration of pharmacy-based distribution of antiviral drugs for the studying construction of strategic national stockpile in some countries.
\end{abstract}

Keywords Urban network · Antiviral drugs · Optimization

Zhanwei Du

duzhanwei0@gmail.com

Limin $\mathrm{Yu}$

yulimin1978@163.com

Chijun Zhang

cjzhang6@163.com

Qing Cai

506183509@qq.com

Zhaohui Li

lizhaohui@nankai.edu.cn

Yuan Bai

baiyuan722@163.com

1 School of Management Science and Information Engineering, Jilin University of Finance and Economics, Changchun 130117, China
2 Jilin Province Key Laboratory of Fintech, Jilin University of Finance and Economics, Changchun 130117, China

3 Jilin Big Data Research Center for Business, Jilin University of Finance and Economics, Changchun 130117, China

4 Department of Integrative Biology, University of Texas at Austin, Austin 78705, USA

5 School of Computer Science and Engineering, Nanyang Technological University, Singapore, Singapore

6 Shandong Agriculture and Engineering University, Jinan 250100, China

7 College of Computer and Control Engineering, Nankai University, Tianjin 300071, China

8 College of Computer Science and Software Engineering, Shenzhen University, Shenzhen 518060, China 


\section{Introduction}

In the early twenty-first century, people have already suffered several global epidemic influenzas [such as pandemic H1N1 in 2009 (Centers for Disease Control and Prevention 2010) and H7N9 in 2013 (Centers for Disease Control and Prevention 2014)] with enormous life and property losses. The risk of a future pandemic influenza outbreak is expected to increase in the coming decades (US Agency for International Development 2016), resulting in health burdens to infected individuals, societal economic burdens to their families and the whole society. Once there is an influenza pandemic, public health officers will take both antiviral drugs and vaccines as the primary response strategies to control influenza pandemics efficacious (Couch 1999; Monto 2006; Bai et al. 2017; Du et al. 2015; Zhanwei and Yongjian 2015; Du and Bai 2018). However, the developing and delivering vaccines for diseases may need several months (Bregu et al. 2011), antiviral drugs are thus particularly critical and taken as the major pharmaceutical intervention in the early stage of the pandemic influenza outbreak.

In preparation for coming influenza pandemics, the US governments have maintained a large Strategic National Stockpile (SNS) of antiviral drugs as a major component in their pandemic response plans (US Department of Health and Human Services 2005). To limit strain on hospitals and improve convenience, the commercial pharmacies have been taken as antiviral drug-dispensing partners in some state pandemic response plans [such as Virginia (Virginia Department of Health 2009) and Louisiana (Office of Public Health 2011)]. It is thus urgent to optimize the current response strategy for the dispensing of antiviral drugs. The goal of this paper is to explore an optimization method to assign a given amount of antiviral drugs to the chosen location of dispensing points (such as pharmacies) within a city, to match practical demand with supply (Fig. 1).

In the existing research of optimizing pharmacy-based response strategy, there are a number of researches (Canoyer 1946; Brown 1992; Hernandez and Bennison 2000), studying the social factors (such as traffic and consumption environment) that a consumer might consider when choosing a pharmacy to buy medicines. The pharmacy site selection problem can be considered as the traditional location problem in theory of combinatorial optimization. However, the site location selection becomes increasingly complex once we consider it additionally from the social perspective. Specifically, with the existing pharmacies, an optimization problem is how to assign the limited dose of drug into different pharmacies in order to benefit the society best. In the related existing mathematical models, they are mostly data driven to help the government to choose pharmacies for antiviral drugs through 0-1 integer linear programming (Singh et al. 2015). In their modeling, the single objective is to maximize the access of the target population to pharmacies. However, there are substantial inevitable but essential social scales needed to be considered:

- The access is investigated via the distance in many retrospective studies (Singh et al. 2015). However, except the geographical condition, the access, denoted as the tendency of pharmacy visit by people, is additionally influenced by people's willingness, depending on social factors [such as the pharmacy reputation (Tao and Liu 2015)].

- The social unbalance for management areas should be considered (Singh et al. 2015). The small management areas should also be assigned, while the previous singleobjective modeling process cannot reflect the balance between the multiple optimization objectives (access and social unbalance).

- The spatial unbalance, as the pharmacy-to-pharmacy distance, should be also taken into consideration. Two
Fig. 1 Drug distribution frame based on practical demand. Antiviral drugs can be accessed by most population, distributed on sparsely populated areas and pharmacies who are geographically far apart

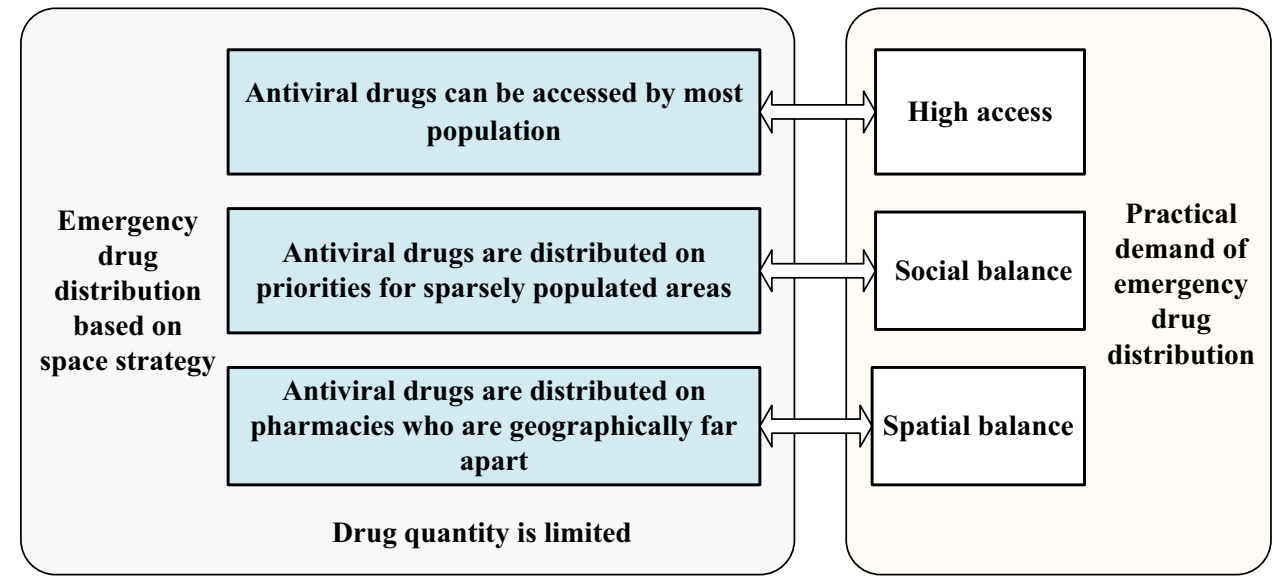


close pharmacies may be chosen at the same time. While in reality, the distribution of pharmacies should be in a sparse spatial network (edges with long pharmacy-topharmacy distance) to supply more opportunities for people.

- The resource unbalance, as the quantitative analysis, is necessary, especially important in resource allocation during the outbreak of pandemics. The amounts of antiviral drugs assign to each chosen pharmacy should in a reasonable range.

To sum up, we model the above considerations in the network-based frame, taking pharmacies as nodes, edges weighted by nodes' distance. The concept of group infers the cluster of nodes located in a same management area. In addition, to maximize the people's access to the pharmacies, there are at least three other objectives needed to be considered: first is the social unbalance from the group perspective. Each target areas, even small, should be assigned to antiviral drugs. Second is the spatial unbalance from the edge perspective. The chosen pharmacies should not be too close to each other. The last is the resource unbalance from the node perspective. The volume of antiviral drug assigned to a pharmacy should be in larger than a certain threshold, to avoid too many administrative works for the trivial assignment, and smaller than another threshold, to limit the unpractical huge amount of drugs assigned.

Thus to assign the optimized amount of antiviral drug for each pharmacy, we propose a network-based model by maximizing multiple objectives (access, social unbalance, spatial unbalance, and resource unbalance). Here the access is based on a willingness-to-travel model to reflect people's tendency to a pharmacy according to its distance and reputation. As for social unbalance, it is modeling with $L_{12}$ norm to make even small areas have antiviral drugs supplied. Besides, to meet the spatial unbalance between any two pharmacies, the concept of network lasso (Hallac et al. 2015) is introduced here to constrain the choices of pharmacies. For the resource unbalance, we limit the volume range of antiviral drugs designed for a pharmacy. Taking the city of Shanghai in 2015 as an example, we use this model to optimize the commercial pharmacy distribution network, given a fixed amount of antiviral drugs. For example of five pharmacies (as plotted in Fig. 2), the 1st and 2nd are in the blue district, and the other three are in the orange district. When we target the optimized group of pharmacies, the balance is studied in terms of meet multiple objectives (access, social unbalance and sparsely spatial distribution) by considering their popularity, distances and sparsity. This research can supply an initial exploration of pharmacy-based distribution of antiviral drugs for the studying construction of strategic national stockpile in China's Twelfth 5-year plan (Development Center of Science and Technology of Chinese Pharmaceutical Association 2014), as well as the diverse applications in computer science ( $\mathrm{Li}$ et al. 2018a; Liu et al. 2018a, b, 2016; Cui et al. 2016).
Fig. 2 Spatial map of districts and pharmacies in Shanghai. a From the group level, Shanghai is divided into different districts represented by colors. For example of five pharmacies, the 1st and 2nd are in the blue district, and the other three are in the orange district. When we target the optimized group of pharmacies, the balance is studied in terms of meet multiple objectives (access, social unbalance and sparsely spatial distribution) by considering their popularity, distances and sparsity. b From the node level, the sites of pharmacies considered here are shown with circles. (Color figure online) (a) Group-Level

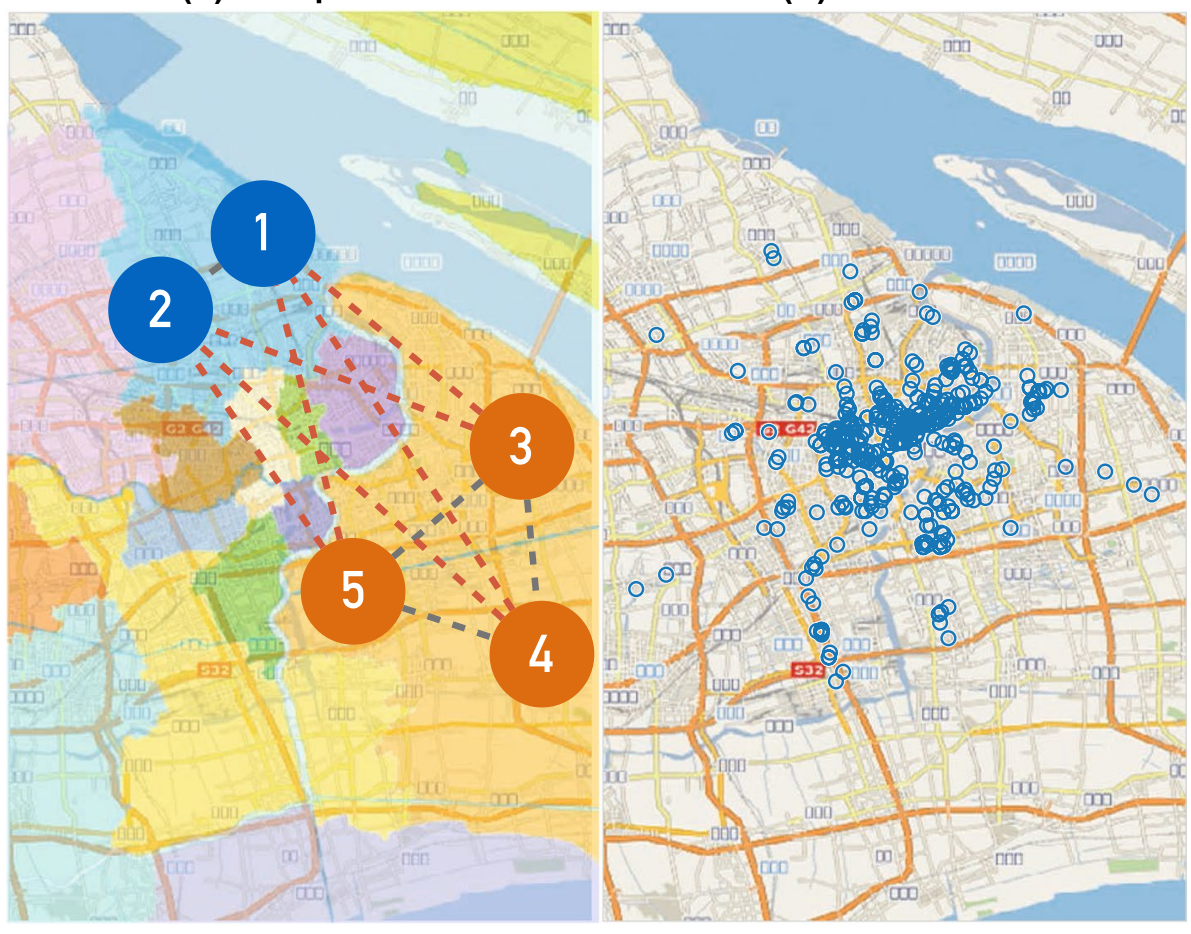




\section{Methods}

\subsection{Data}

\subsubsection{Subway}

To describe the population distribution of Shanghai, we use the travel smartcard dataset (collected by Shanghai Public Transportation Card Co. Ltd) in Shanghai, China during 30 days in April 2015. This dataset involves 313 subway stations, 11 million individuals and 123 million events of trips. Once people travel through the subway stations by their smart card, when and where this event happens will be recorded automatically (Seasson Information Technology Co. Ltd 2015). With these records, we mainly consider the volume of check-in passengers for each station to reveal the population distribution.

\subsubsection{Pharmacy}

To get the spatial information of pharmacies in Shanghai, we use the API of Baidu Map to get their names and spatial positions (Baidu Inc. 2014). Specifically, there are around 5600 pharmacies, in which there are 2036 pharmacies marked by 11 famous brands (which have more than 50 pharmacies in Shanghai). As for their ratings of popular, they can be found on the website of Dianping, one of China's leading O2O platforms for urban and lifestyle services (Dianping 2016).

With this information of both network and individual nodes shown in Fig. 2, our aim is to find the optimized group of pharmacies to meet multiple objectives (access, social unbalance and sparsely spatial distribution).

\subsection{Willingness-to-travel model}

We use the gravity model (Zipf 1946; Barthélemy 2011; Erlander and Stewart 1990) to estimate the effect of distances on individuals' willingness to travel in Shanghai to get antiviral drugs from pharmacies. Besides, the influence of distances on people's willingness, pharmacies' own social profiles (such as reputation and scale) also show obvious effect (Tao and Liu 2015). Such the probability of people's willingness to the $i$ th pharmacy can be modeled by the following equation: $p(i)=\sum_{j} p_{i j} S_{i} R_{i}$ Here $p_{i j}$ is the effect of distances on the willingness by gravity model. $S_{i}$ denotes the scale of the $i$ th pharmacy, as the number of drug pharmacys in the brand which the $i$ th drug pharmacy belongs. $R_{i}$ denotes the reputation of the $i$ th drug pharmacy, as the rating of popular by individuals.

\subsection{Optimization model}

We mainly consider three items for the multi-objectiveness of access, social unbalance, spatial unbalance and resource unbalance.

1. The access is used to reflect people's tendency to a pharmacy according to its distance and popularity. For a pharmacy, the tendency is proportional to the number of people living around and the popularity (such as the scale and reputation) of this pharmacy. Specifically, let $\alpha$ be the access measure of the assigned volume of drug, denoted as $x_{i}$, for the $i$ th drug pharmacy. We set $\alpha$ with element $\alpha_{i}=\sum_{j}$ pop $_{j} p_{i j} S_{i} R_{i}$. Here pop $_{j}$ is the population for the $j$ th station, $S_{i}$ is the scale of the $i$ th drug pharmacy and $R_{i}$ denotes the reputation of the $i$ th pharmacy. Let $p_{i j}=\frac{1}{r_{i j}^{2}}$, where $r_{i j}$ represents the distance between the $j$ th station and the $i$ th drug pharmacy.

2. As for the social unbalance, $l_{12}$ norm is commonly used as the exclusive Lasso for multi-view feature selection (Bach et al. 2012), which is suitable for us to balance the resources in different management areas. Thus no area is assigned without resources. The only difference between areas is the dose of the drug distributed.

3. To meet the request of spatial unbalance as the sparsely spatial distribution, a network-lasso penalty operator $D$ (Hallac et al. 2015) is introduced here, which is used to describe the relationships between two nodes. In our case, the closer two chosen pharmacies are, the larger the penalty tends to be.

4. The resource unbalance is modeled by constraining the volume range of antiviral drugs designed for a pharmacies. We limit $x_{i}$ in the range of $\left[\xi, \alpha_{i}\right]$,

To sum up, with the above multi-objectiveness, we propose the following optimization model for the pharmacy-based distribution of antiviral drugs:

$$
\begin{array}{ll}
\max & \sum_{i} \alpha_{i} x_{i}-\lambda\|x\|_{12}-\mu D \\
\text { s.t. } & \alpha_{i}=\sum_{j} \text { pop }_{j} p_{i j} S_{i} R_{i} \\
& \sum_{i} x_{i}=M_{m e d} \\
& x_{i} \in\left[\xi, \alpha_{i}\right] \\
& \|x\|_{12}=\sum_{w \in K}\left(\sum_{m \in w} x_{m}^{w}\right)^{2} \\
D=\sum_{i, k} \frac{1_{x_{i} \leq \xi} 1_{x_{k} \leq \xi}}{d_{i k}}
\end{array}
$$


Here, $x_{i}$ represents the volume of drug assignment for the $i$ th drug pharmacy. $D$ is the network-lasso penalty operator, constraining each pair of pharmacies. $\alpha_{i}$ is the access influence and also the upper bound of $x_{i}$. $\lambda$ infers the influence of social unbalance. $\mu$ denotes the influence of spatial influence. $\|x\|_{12}$ denotes $l_{12}$ norm of groups of drug pharmacies. 1 . is the indicator function. $1_{x_{i}}$ denotes whether $x_{i}$ is larger than $\xi$ drug pharmacy. $x_{m}^{w}$ denotes the volume of drug assignment for the $m$ th station in the $w$ th management area among the $K$ management areas. $d_{i k}$ means the distance between the $i$ th drug pharmacy and the $k$ th drug pharmacy. $\xi$ is the lower bound of $x_{i}$. $M_{\text {med }}$ denotes the total dose of drugs.

The above model can be considered as a constrained nonlinear multivariable function, which can use Hessianbased (the second derivatives of the Lagrangian) method by a dense quasi-Newton approximation (Haelterman et al. 2009). Besides, to show the social and spatial unbalances intuitively, we consider the following two rates to measure them respectively:

- Rate of spatial relations within $1 \mathrm{~km}$, which denotes the ratio between the number of pharmacies pairs (whose spatial distance is less than $1 \mathrm{~km}$ ) and the total number of pharmacies pairs.

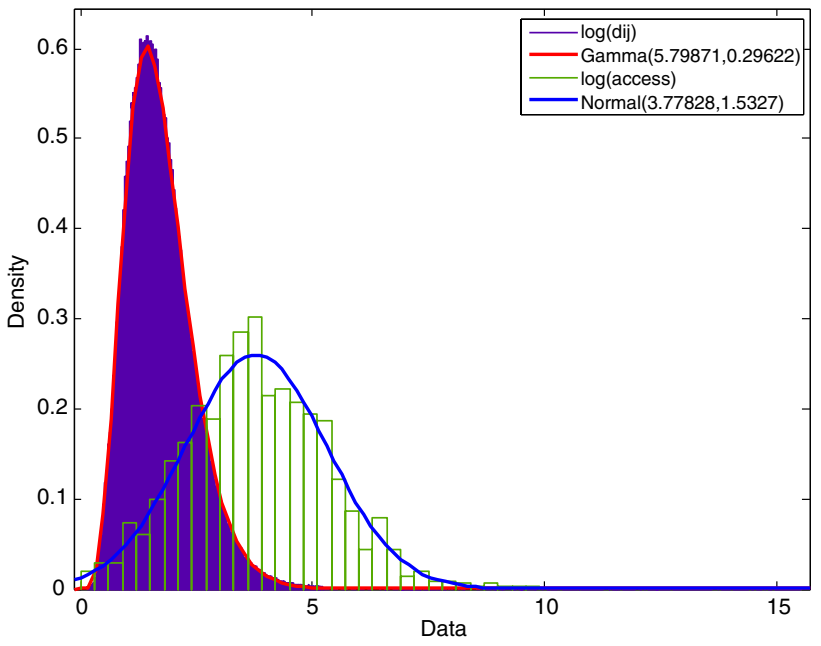

Fig. 3 Density distribution of access for each pharmacy and distance between two pharmacies

- Coverage rate of districts, which means the ratio between the number of districts (who have at least one pharmacy assigned) and the total number of districts.
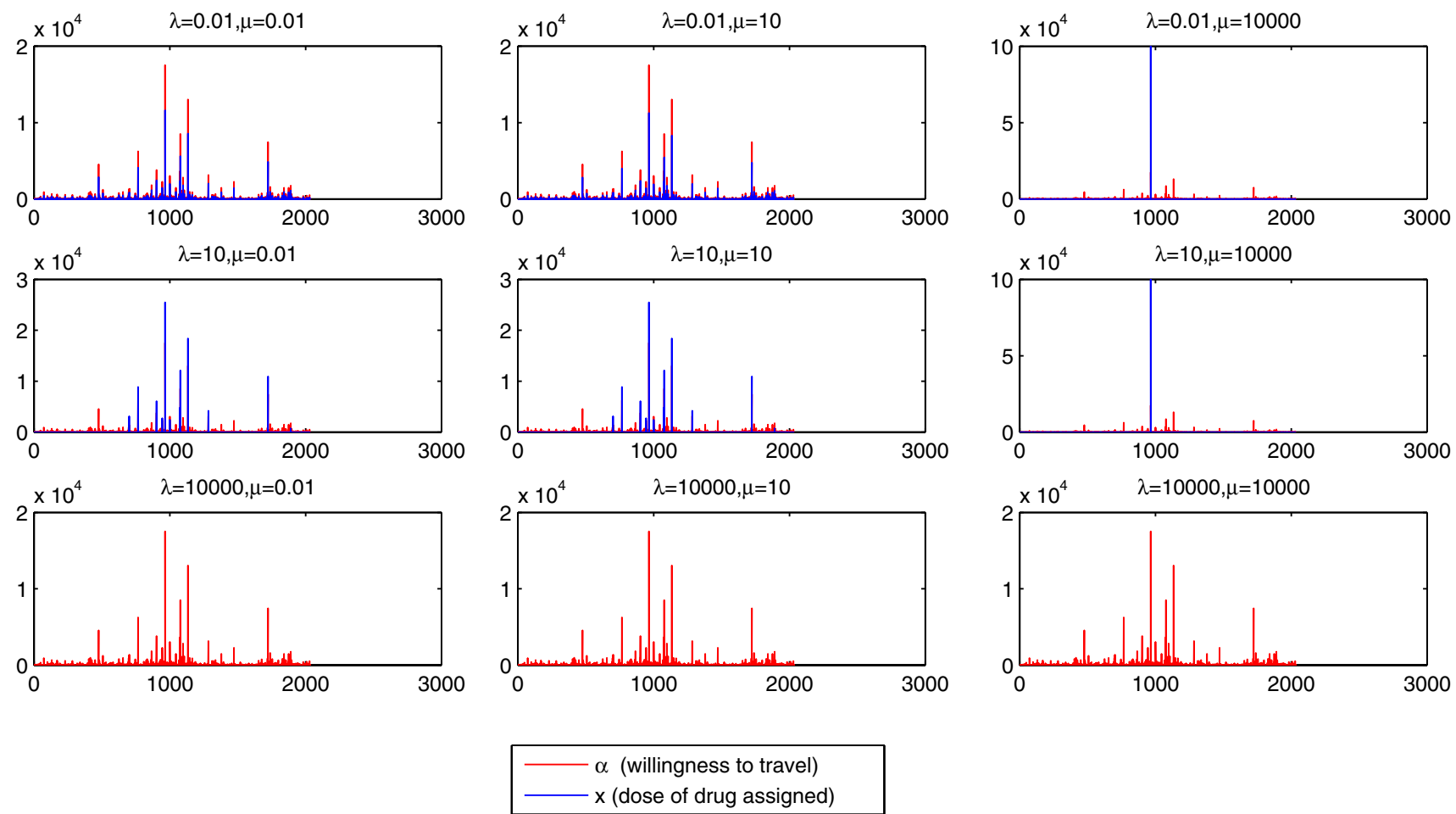

Fig. 4 Dynamics of pharmacies' access with varying lambda $(\lambda)$ and $\mathrm{mu}(\mu)$ from 0.01 to 10,000 . When lambda and mu are small in subgraph a, many pharmacies are chosen for drug assignment. As for the increasing mu and lambda, the drugs tend to focus on a single pharmacy or different pharmacies in dynamic districts 


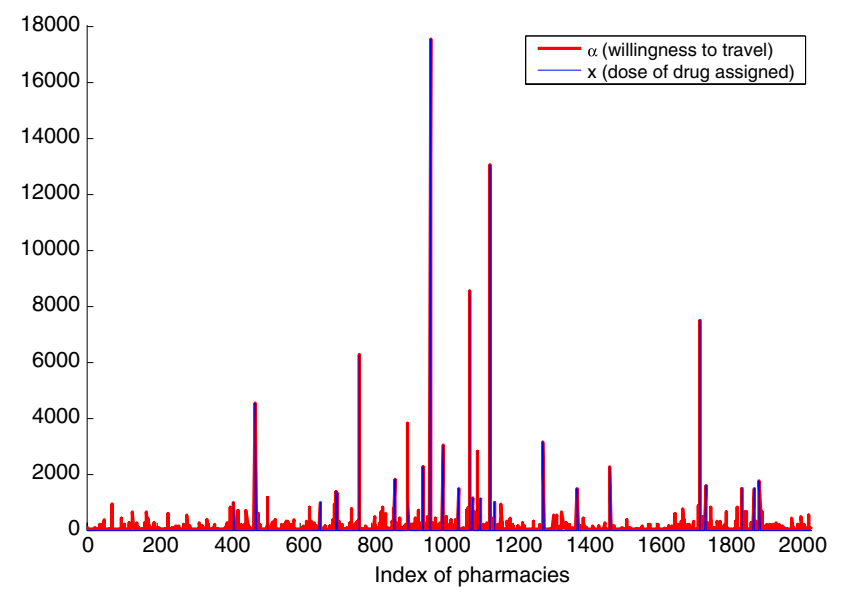

Fig. 5 Pharmacies' antiviral drug distribution without considering social and spatial unbalances. The $\mathrm{x}$-axis is indexed by the pharmacies. Comparing with $\alpha$, the top 29 pharmacies with highest access are chosen

\section{Results}

During early 2016, there are 2036 pharmacies in Shanghai from 11 famous in 15 administrative districts. To characterize the properties of pharmacies' network, we start by analyzing the density distribution of access and distance, shown in Fig. 3. To be specific, the density distribution of logarithmic access follows the Gamma distribution, while the logarithmic distance follows the Normal distribution. These distributions tell us that the pharmacies with high or low access are in the minority, the same with the distances.

Next, we begin to evaluate the optimization model for the pharmacy-based distribution of antiviral drugs. Assuming there are $10^{5}$ dose of antiviral drugs for assignment, which is more than any single pharmacy's access, but not enough for the full coverage of all pharmacies. We estimate the spatial network of pharmacies shown in Figs. 4 and 6 by varying the influence of social unbalance and spatial unbalance, given resource unbalance as a fixed threshold for the minimum of

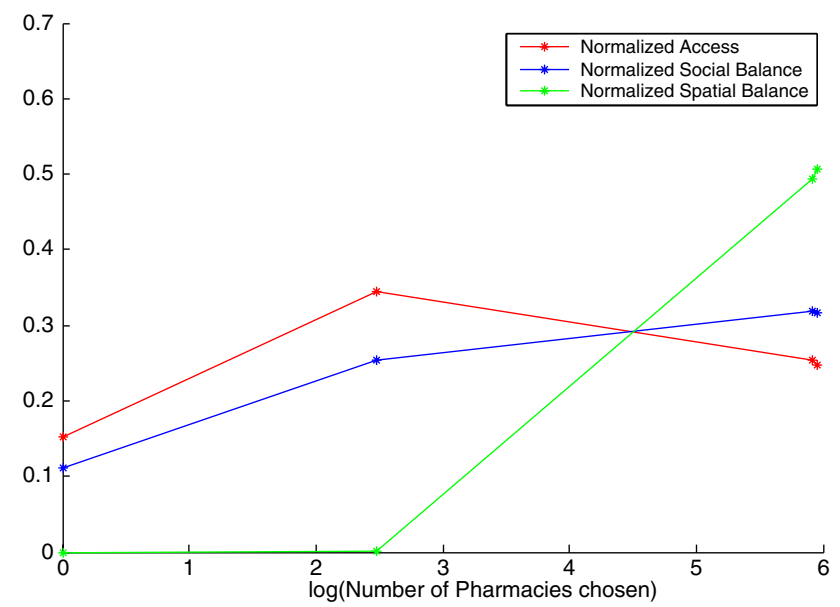

Fig. 7 Normalized Objectives with dynamic number of pharmacies chosen. Taking ID. $\mathrm{a}-\mathrm{f}$ as the representation of Table 1 , the normalized objectives (access, social and spatial unbalance) are shown. When there are few pharmacies, the normalized social unbalance shows more influence. Once the number of pharmacies exceed its peak, the normalized spatial unbalance tends to show its influence

pharmacies' drug for assignment. With increasing influence of social unbalance (represented by $\lambda$ ), decreasing number of pharmacies are assigned with drugs across different districts, showing the social unbalance to avoid the unbalance assigned resources of across management areas. In contrast, with the increasing spatial influence (denoted by $\mu$ ), the doses of drugs tend to be in only one pharmacy, which has the highest access influence. Comparing with the traditional single objective (where $\lambda$ and $m u$ are all 0 ), the drug distribution of pharmacies is fixed, as shown in Figs. 5 and 6. Many pharmacies locate at the city center, and close to each other.

We additionally explore the relationship between the number of chosen pharmacies and the desirable objectives (access, social and spatial unbalances) achieved, shown in Table 1 and Fig. 7. The social and spatial unbalance are both increasing with the number of chosen pharmacies. While for

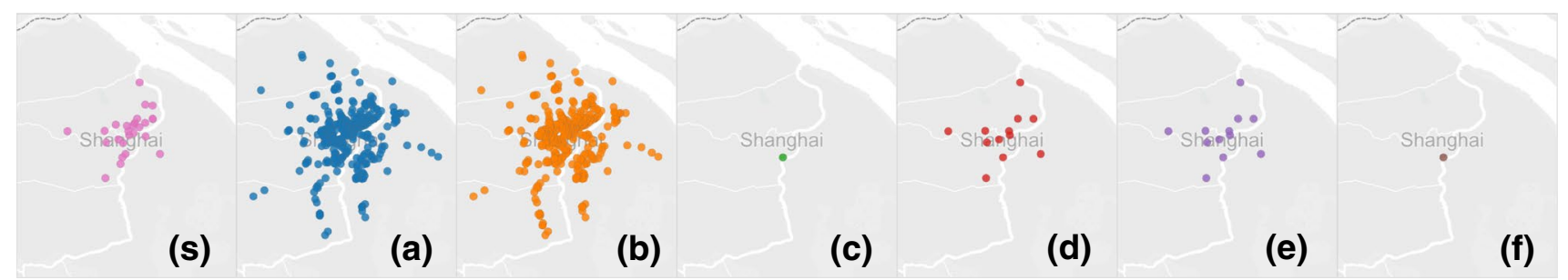

Fig. 6 Spatial map of chosen pharmacies for varying $\lambda$ and $\mu$. Each blue point denotes a pharmacy chosen. The indexes of subgraph are corresponding to the IDs in Table 1 
Table 1 Items involved in the optimization model, corresponding with Fig. 4

\begin{tabular}{|c|c|c|c|c|c|c|c|c|}
\hline ID & $\begin{array}{l}\text { Pharmacy } \\
\text { number }\end{array}$ & Access & Social unbalance & Spatial unbalance & $\lambda$ & $\mu$ & $\begin{array}{l}\text { Coverage rate of } \\
\text { districts }(\%)\end{array}$ & $\begin{array}{l}\text { Rate of spatial } \\
\text { relations within } \\
1 \mathrm{~km}\end{array}$ \\
\hline s & 29 & $7.60 \mathrm{E}+08$ & $1.14 \mathrm{E}+09$ & $1.07 \mathrm{E}+05$ & 0 & 0 & 81.3 & $0.49 \%$ \\
\hline $\mathrm{a}$ & 371 & $5.15 \mathrm{E}+08$ & $8.76 \mathrm{E}+08$ & $8.83 \mathrm{E}+06$ & 0.01 & 0.01 & 87.5 & $0.01 \%$ \\
\hline $\mathrm{b}$ & 387 & $5.01 \mathrm{E}+08$ & $8.72 \mathrm{E}+08$ & $9.04 \mathrm{E}+06$ & 0.01 & 10 & 87.5 & $0.01 \%$ \\
\hline $\mathrm{c}$ & 1 & $3.07 \mathrm{E}+08$ & $3.07 \mathrm{E}+08$ & 0 & 0.01 & $1.00 \mathrm{E}+04$ & 6.3 & - \\
\hline d & 12 & $6.98 \mathrm{E}+08$ & $6.96 \mathrm{E}+08$ & $1.92 \mathrm{E}+03$ & 10 & 0.01 & 75.0 & $0.00 \%$ \\
\hline $\mathrm{e}$ & 12 & $6.98 \mathrm{E}+08$ & $6.96 \mathrm{E}+08$ & $1.92 \mathrm{E}+03$ & 10 & 10 & 75.0 & $0.00 \%$ \\
\hline $\mathrm{f}$ & 1 & $3.07 \mathrm{E}+08$ & $3.07 \mathrm{E}+08$ & 0 & 10 & $1.00 \mathrm{E}+04$ & 6.3 & - \\
\hline $\mathrm{g}$ & 0 & 0 & 0 & 0 & $1.00 \mathrm{E}+04$ & 0.01 & 0.0 & - \\
\hline h & 0 & 0 & 0 & 0 & $1.00 \mathrm{E}+04$ & 10 & 0.0 & - \\
\hline i & 0 & 0 & 0 & 0 & $1.00 \mathrm{E}+04$ & $1.00 \mathrm{E}+04$ & 0.0 & - \\
\hline
\end{tabular}

The item 'ID' denotes the index of sub-graphs in Fig. 4, and the same as the other items

the access, there is a peak. Too little or too many pharmacies are leading the access influence decreasing. Specifically, there are 29 pharmacies needed with high social unbalance (covering $81 \%$ districts) by the traditional single-objective method of access. In the contrast, only 12 pharmacies are needed with similar access ability but smaller social unbalance (covering $75 \%$ districts). Or more pharmacies are assigned with covering $87 \%$ districts. As for the spatial unbalance, the rates of spatial relations within $1 \mathrm{~km}$ in the proposed optimization model are still improved in conditions of less or more pharmacies assigned. From this, we can see the trade off among social unbalance, spatial unbalance and the access to the dynamic number of pharmacies assigned.

\section{Discussion}

Increasing governments take the pharmacies in their plans for the dispensing of antiviral drugs. Whether and how much this strategy can benefit the society is needed to study, especially for the constructing strategic national stockpile. In this paper, we study an optimization method to assign a given amount of antiviral drugs to pharmacies within a city. A network-based method is proposed by maximizing multiple objectives (access, social unbalance, spatial unbalance and resource unbalance). We can see how these objectives influence the number of pharmacies chosen. The improvements of social and spatial unbalance are in the cost of access.

Our studied results can help deepen the understanding of antiviral drug distribution in the network of pharmacies within cities by evaluating this interesting strategy in different perspectives. The methods used might be specific, but the questions raised can be valuable on their own and motivate research using other types of data (Lin et al. 2017a, b; He et al. 2017; Yuan et al. 2017; Li et al. 2018b, c; Cai et al. 2017; Shen et al. 2018; Jia et al. 2017; Liu et al. 2018c; Huang et al. 2018; Wang et al. 2018).

Acknowledgements The authors are grateful for the support of National Social Science Foundation of China no. 16BGL180.

\section{References}

Bach F, Jenatton R, Mairal J, Obozinski G (2012) Optimization with sparsity-inducing penalties. Foundations and Trends ${ }^{\circledR}$. Mach Learn 4(1):1-106

Bai Y, Yang B, Lin L, Herrera JL, Du Z, Holme P (2017) Optimizing sentinel surveillance in temporal network epidemiology. Sci Rep $7(1): 4804$

Baidu Inc. (2014) Javascript api for Baidu map. http://lbsyun.baidu .com/index.php?title=jspopular. Accessed 30 Dec 2014

Barthélemy M (2011) Spatial networks. Phys Rep 499(1):1-101

Bregu M, Draper SJ, Hill AV, Greenwood BM (2011) Accelerating vaccine development and deployment: report of a royal society satellite meeting. Philos Trans R Soc Lond B Biol Sci 366(1579):2841-2849

Brown S (1992) Retail location: a micro-scale perspective. Avebury

Cai J, Wang Y, Liu Y, Luo JZ, Wei W, Xu X (2017) Enhancing network capacity by weakening community structure in scale-free network. Future Gener Comput Syst. https://doi.org/10.1016/j. future.2017.08.014

Canoyer HG (1946) Selecting a store location 56. US Govt. print. off

Centers for Disease Control and Prevention (2010) The 2009 h1n1 pandemic: summary highlights, April 2009-April 2010. Official Online Article Published by the Centers for Disease Control, 4

Centers for Disease Control and Prevention (2014) Background and summary of human infection with influenza A (h7n9) virus. http:// www.who.int/influenza/human_animal_interface. Accessed 1 Jul 2017 
Couch RB (1999) Measures for control of influenza. Pharmacoeconomics 16(1):41-45

Cui B, Liu Z, Wang L (2016) Key-aggregate searchable encryption (kase) for group data sharing via cloud storage. IEEE Trans Comput 65(8):2374-2385

Development Center of Science and Technology of Chinese Pharmaceutical Association (2014) National twelfth-five-yearplan seminar of major issues: research of the construction of national emergency response system of drug. http://www.gov. cn/zwgk/2012-02/13/content_2065197.htm. Accessed 1 Jul 2017

Dianping (2016) Javascript api for baidu map

Du Z, Bai Y (2018) Detecting the impact of public transit on the transmission of epidemics. arXiv:180109333

Du Z, Yang Y, Ma C, Bai Y (2015) Modelling individual negative emotion spreading process with mobile phones. In: AAAI, pp 4156-4157

Erlander S, Stewart NF (1990) The gravity model in transportation analysis: theory and extensions, vol 3. VSP, Utrecht, The Netherlands

Haelterman R, Degroote J, Van Heule D, Vierendeels J (2009) The quasi-newton least squares method: a new and fast secant method analyzed for linear systems. SIAM J Numer Anal 47(3):2347-2368

Hallac D, Leskovec J, Boyd S (2015) Network lasso: clustering and optimization in large graphs. In: Proceedings of the 21th ACM SIGKDD international conference on knowledge discovery and data mining. ACM, pp 387-396

He P, Deng Z, Gao C, Wang X, Li J (2017) Model approach to grammatical evolution: deep-structured analyzing of model and representation. Soft Comput 21(18):5413-5423

Hernandez T, Bennison D (2000) The art and science of retail location decisions. Int J Retail Distrib Manag 28(8):357-367

Huang Y, Li W, Liang Z, Xue Y, Wang X (2018) Efficient business process consolidation: combining topic features with structure matching. Soft Comput 22(2):645-657

Jia B, Liu S, Guan Y, Li W, Ren W (2017) The fusion model of multidomain context information for the internet of things. Wireless Commun Mob Comput

Li R, Wu X, Liu H, Wu J, Yin M (2018b) An efficient local search for the maximum edge weighted clique problem. IEEE Access 6:10743-10753

Li Y, Wang G, Nie L, Wang Q, Tan W (2018c) Distance metric optimization driven convolutional neural network for age invariant face recognition. Pattern Recognit 75:51-62

Li B, Huang Y, Liu Z, Li J, Tian Z, Yiu SM (2018) Hybridoram: practical oblivious cloud storage with constant bandwidth. Inf Sci. https ://doi.org/10.1016/j.ins.2018.02.019

Lin W, Xu S, He L, Li J (2017a) Multi-resource scheduling and power simulation for cloud computing. Inf Sci 397:168-186

Lin W, Xu S, Li J, Xu L, Peng Z (2017b) Design and theoretical analysis of virtual machine placement algorithm based on peak workload characteristics. Soft Comput 21(5):1301-1314

Liu Z, Chen X, Yang J, Jia C, You I (2016) New order preserving encryption model for outsourced databases in cloud environments. J Netw Comput Appl 59:198-207
Liu Z, Huang Y, Li J, Cheng X, Shen C (2018a) Divoram: towards a practical oblivious ram with variable block size. Inf Sci 447:1-11

Liu Z, Li T, Li P, Jia C, Li J (2018b) Verifiable searchable encryption with aggregate keys for data sharing system. Future Gener Comput Syst 78:778-788

Liu Z, Wu Z, Li T, Li J, Shen C (2018) GMM and CNN hybrid method for short utterance speaker recognition. IEEE Trans Ind Inform. https://doi.org/10.1109/TII.2018.2799928

Monto AS (2006) Vaccines and antiviral drugs in pandemic preparedness. Emerg Infect Dis 12(1):55

Office of Public Health (2011) State of louisiana department of health and hospitals. Pandemic in uenza guidance annex 4: antiviral

Seasson Information Technology Co. Ltd (2015) Shanghai open data apps. http://soda.datashanghai.gov.cn/. Accessed 1 Jul 2016

Shen J, Gui Z, Ji S, Shen J, Tan H, Tang Y (2018) Cloud-aided lightweight certificateless authentication protocol with anonymity for wireless body area networks. J Netw Comput Appl. https://doi. org/10.1016/j.jnca.2018.01.003

Singh B, Huang HC, Morton DP, Johnson GP, Gutfraind A, Galvani AP, Clements B, Meyers LA (2015) Optimizing distribution of pandemic influenza antiviral drugs. Emerg Infect Dis 21(2):251

Tao L, Liu J (2015) Understanding self-organized regularities in healthcare services based on autonomy oriented modeling. Nat Comput 14(1):7-24

US Agency for International Development (2016) Emerging pandemic threats. https://www.usaid.gov/what-we-do/global-health/pande mic-influenza-and-other-emerging-threats. Accessed 1 Jul 2017

US Department of Health and Human Services (2005) HHs pandemic influenza plan. https://www.cdc.gov/flu/pdf/professionals/hhspa ndemicinfluenzaplan.pdf/ Accessed 1 Jul 2016

Virginia Department of Health (2009) Antiviral distribution plan:attachment pandemic influenza. http://www.vdh.state.va. us/oep/documents/2009/docs/VDH\%20PanFlu\%20Plan\%20Sup plement\%207\%20-\%20Antiviral\%20Dist\%20\&\%20Use\%209-242009.doc. Accessed 8 Dec 2013

Wang H, Wang W, Cui Z, Zhou X, Zhao J, Li Y (2018) A new dynamic firefly algorithm for demand estimation of water resources. Inf Sci 438:95-106

Yuan C, Li X, Wu QJ, Li J, Sun X (2017) Fingerprint liveness detection from different fingerprint materials using convolutional neural network and principal component analysis

Zhanwei D, Yongiian Y (2015) Semi-automatic remote medicine monitoring system of mobile users. China Commun 12(11):1-9

Zipf GK (1946) The p 1 p 2/d hypothesis: on the intercity movement of persons. Am Sociol Rev 11(6):677-686

Publisher's Note Publisher's Note Springer Nature remains neutral with regard to jurisdictional claims in published maps and institutional affiliations 